\title{
Conceptions de la diversité ethnoculturelle dans la formation en enseignement au Québec : un exercice de mise en visibilité des rapports sociaux de race \\ Perceptions of ethnocultural diversity in teacher training in Quebec: An exercise in increasing the visibility of race relations
}

\section{Concepciones de la diversidad etnocultural en la formación magisterial en Quebec: el empleo de la visibilidad de las relaciones sociales de raza}

\author{
Julie Larochelle-Audet
}

Volume 46, numéro 2, automne 2018

Pluralisme, équité et rapports ethniques dans la formation du personnel des milieux éducatifs

URI : https://id.erudit.org/iderudit/1055562ar

DOI : https://doi.org/10.7202/1055562ar

Aller au sommaire du numéro

Éditeur(s)

Association canadienne d'éducation de langue française

ISSN

1916-8659 (numérique)

Découvrir la revue

\section{Citer cet article}

Larochelle-Audet, J. (2018). Conceptions de la diversité ethnoculturelle dans la formation en enseignement au Québec : un exercice de mise en visibilité des rapports sociaux de race. Éducation et francophonie, 46(2), 73-91.

https://doi.org/10.7202/1055562ar

\section{Résumé de l'article}

Vingt ans après l'adoption de la Politique d'intégration scolaire et d'éducation interculturelle (Ministère de l'Éducation, 1998), sa transposition dans les programmes de formation initiale en enseignement fait l'objet d'un bilan mitigé (Larochelle-Audet, Magnan, Potvin et D’Arrisso, 2018). Alors que des discours racistes se banalisent dans l'espace public (Potvin, 2008, 2016), les lacunes constatées dans la formation mettent en question les limites de ces orientations pluralistes. Cet article se penche en particulier sur les conceptions et les usages de la notion de diversité ethnoculturelle dans la formation en enseignement, et plus largement dans l'institution éducative québécoise. Il présente les résultats d'une analyse secondaire de données initialement collectées dans le cadre d'une recherche de maîtrise auprès de neuf professeures de trois universités francophones (Larochelle-Audet, 2014a). À partir d'outils conceptuels du féminisme matérialiste, des fragments permettant de voir les rapports sociaux de race sont assemblés de manière à révéler comment ces rapports sont à la fois dissimulés et réifiés par cette notion. Cet exercice de mise en visibilité s'intéresse successivement à la diversité ethnoculturelle comme contenu de formation, comme propriété de certains groupes de personnes et, enfin, comme voile s'inscrivant dans une logique d'effacement du racisme (Dhume, El Massioui et Sotto, 2016).
Tous droits réservés (C) Association canadienne d'éducation de langue française, 2018
Ce document est protégé par la loi sur le droit d'auteur. L’utilisation des services d'Érudit (y compris la reproduction) est assujettie à sa politique d'utilisation que vous pouvez consulter en ligne. 


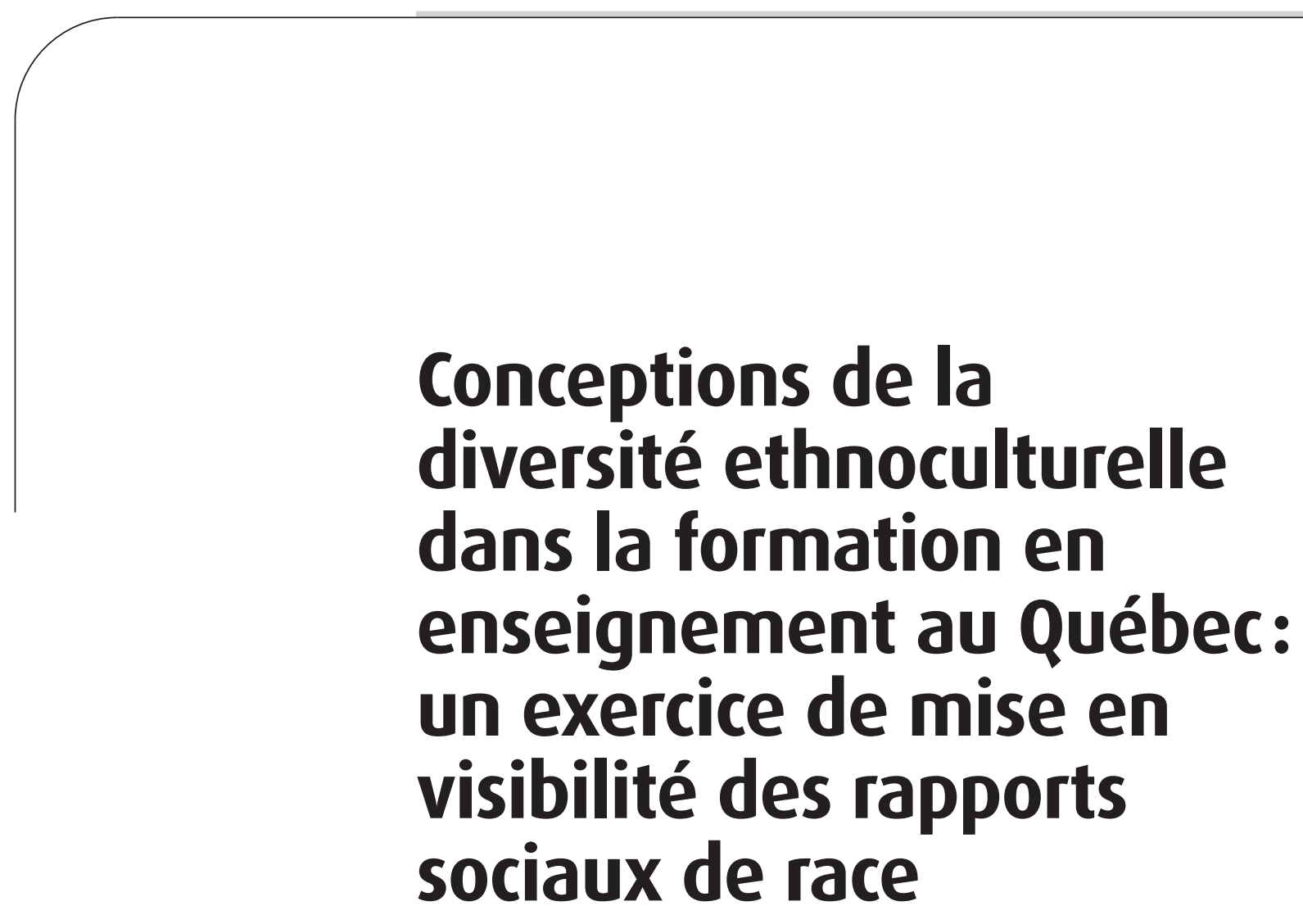

Citer cet article :

LAROCHELLE-AUDET, J. (2018).

Conceptions de la diversité

ethnoculturelle dans la formation

en enseignement au Québec: un

exercice de mise en visibilité des

rapports sociaux de race. Éducation

et francophonie, 46(2), 73-91.

Julie LAROCHELLE-AUDET

Université de Montréal, Québec, Canada

\section{RÉSUMÉ}

Vingt ans après l'adoption de la Politique d'intégration scolaire et d'éducation interculturelle (Ministère de l'Éducation, 1998), sa transposition dans les programmes de formation initiale en enseignement fait l'objet d'un bilan mitigé (Larochelle-Audet, Magnan, Potvin et D'Arrisso, 2018). Alors que des discours racistes se banalisent dans l'espace public (Potvin, 2008, 2016), les lacunes constatées dans la formation mettent en question les limites de ces orientations pluralistes. Cet article se penche en particulier sur les conceptions et les usages de la notion de diversité ethnoculturelle dans la formation en enseignement, et plus largement dans l'institution éducative québécoise. Il présente les résultats d'une analyse secondaire de données initialement collectées dans le cadre d'une recherche de maitrise auprès de neuf professeures de trois universités francophones (Larochelle-Audet, 2014a). À partir d'outils conceptuels 
du féminisme matérialiste, des fragments permettant de voir les rapports sociaux de race sont assemblés de manière à révéler comment ces rapports sont à la fois dissimulés et réifiés par cette notion. Cet exercice de mise en visibilité s'intéresse successivement à la diversité ethnoculturelle comme contenu de formation, comme propriété de certains groupes de personnes et, enfin, comme voile s'inscrivant dans une logique d'effacement du racisme (Dhume, El Massioui et Sotto, 2016).

\section{ABSTRACT}

\section{Perceptions of ethnocultural diversity in teacher training in Quebec: An exercise in increasing the visibility of race relations}

Julie LAROCHELLE-AUDET, University of Montreal, Quebec, Canada

Twenty years after the adoption of the Policy on Educational Integration and Intercultural Education (Department of Education, 1998), its transposition into initial teacher training programs has had mixed reviews (Larochelle- Audet, Magnan, Potvin and D'Arrisso, 2018). While racist discourse is becoming commonplace in the public sphere (Potvin, 2008, 2016), the shortcomings noted in teacher training challenge the boundaries of these multicultural orientations. This article looks specifically at perceptions and usages of the notion of ethnocultural diversity in teacher training, and more broadly in Quebec schools. It presents the results of a secondary analysis of data first collected for a Master's study with nine professors from three French-speaking universities (Larochelle-Audet, 2014a). Using conceptual tools from materialist feminism, fragments that reveal race relations are assembled to show how these relations are both concealed and reified by this notion. This visibility exercise focuses successively on ethnocultural diversity as training content, as the property of certain groups of people and, finally, as a veil for the logic of the elimination of racism (Dhume, El Massioui and Sotto, 2016).

\section{RESUMEN}

\section{Concepciones de la diversidad etnocultural en la formación magisterial en Quebec: el empleo de la visibilidad de las relaciones sociales de raza}

Julie LAROCHELLE-AUDET, Universidad de Montreal, Quebec, Canadá

Veinte años después de la adopción de la Política de integración escolar y de educación intercultural (Ministère de l'Éducation, 1998), su transposición en los programas de formación básica en enseñanza fue objeto de una evaluación desigual (LarochelleAudet, Magnan, Potvin et D’Arrisso, 2018). Mientras que los discursos racistas se banalizan en el espacio público (Potvin, 2008, 2016), las lagunas constatadas en la 
formación cuestionan los limites de dichas orientaciones pluralistas. Este artículo se centra particularmente en las concepciones y usos de la noción de diversidad etnocultural en la formación en enseñanza, y más ampliamente en la institución educativa quebequense. Presenta los resultados de un análisis secundario de datos inicialmente compilados en el cuadro de una investigación de maestría entre nueve profesoras de tres universidades francófonas (Larochelle-Audet, 2014a). A partir de las herramientas conceptuales del feminismo materialista, algunas partes permiten ver que las relaciones sociales de raza se ensamblan de una manera que muestra como dichas relaciones se disimulan y reifican en dicha noción. Este empleo de la visibilidad se interesa de manera sucesiva a la diversidad etnocultural como contenido de la formación, como propiedad de ciertos grupos de personas y finalmente como velo inscrito en una lógica de reducción del racismo (Dhume, El Massioui et Sotto, 2016).

\section{INTRODUCTION}

Il y a vingt ans, la Politique d'intégration scolaire et d'éducation interculturelle appelée après politique de 1998 - faisait de la formation du personnel scolaire l'une des conditions essentielles à la mise en œuvre d'un système scolaire répondant aux principes de l'éducation interculturelle, soit «savoir vivre ensemble dans une société francophone, démocratique et pluraliste» (Ministère de l'Éducation, 1998, p. 23). Une de ses orientations prescrit le développement d'une solide formation initiale et continue afin de préparer le personnel scolaire à «relever les défis éducatifs liés à la diversité ethnoculturelle, linguistique et religieuse de la société québécoise» (Ministère de l'Éducation, 1998, p. 32).

Vingt ans après l'adoption de cette politique, cette orientation fait l'objet d'un bilan mitigé (Larochelle-Audet et al., 2018). En dépit de la politique de 1998, la prise en compte de la diversité ethnoculturelle ne figure pas explicitement parmi les douze compétences professionnelles du référentiel élaboré par le ministère de l'Éducation du Québec (2001a, 2001b) orientant les programmes de formation initiale à l'enseignement ${ }^{1}$ (Larochelle-Audet, Borri-Anadon et Potvin, 2016; Potvin et al., 2015). Un portrait de la formation de la relève enseignante sur cette question dans les universités

1. Pour être accrédités, les programmes offerts par les universités québécoises doivent se conformer aux orientations ministérielles et faire l'objet d'une évaluation périodique par le Comité d'agrément des programmes de formation à l'enseignement. Chaque compétence prescrite par le ministère doit être développée et évaluée en contexte de formation (cours et stages). 
québécoises montre qu'une partie du personnel enseignant obtient le diplôme ${ }^{2}$ sans avoir eu de cours à ce sujet (Larochelle-Audet, Borri-Anadon, Mc Andrew et Potvin, 2013). Si le nombre de cours s'est globalement accru durant cette période, ils se sont principalement concentrés dans les programmes visant à former le personnel enseignant qui travaillera auprès d'élèves immigrants ou en processus d'apprentissage de la langue d'enseignement (Borri-Anadon, Larochelle-Audet, Potvin et Mc Andrew, 2014).

Alors que des discours racistes allant jusqu'au désir d'expulser les "Autres» se sont vus légitimés politiquement ces dernières années (Potvin, 2008, 2016), cet article met en question les limites de ces orientations pluralistes, déjà mises en lumière quelques années auparavant par Donatille Mujawamariya et Mirela Moldoveanu pour le Canada (Moldoveanu et Mujawamariya, 2007; Mujawamariya, 2006; Mujawamariya et Moldoveanu, 2006). Il approfondit en particulier les conceptions et les usages de la notion de diversité ethnoculturelle ayant émergé dans le cadre de ma recherche de maîtrise ${ }^{3}$, qui visait à comprendre comment les acteurs des universités québécoises contribuent à l'institutionnalisation de la diversité ethnoculturelle dans les programmes de formation initiale à l'enseignement (Larochelle-Audet, 2014a, 2014b). À partir d'une analyse secondaire de ces données, je délaisse le point de vue institutionnel initialement adopté afin d'explorer les rapports sociaux de race à partir d'outils conceptuels développés par des féministes matérialistes. C'est dans cette perspective que j'utilise le concept de «rapports sociaux de race» défini ci-après.

Après avoir présenté l'ancrage conceptuel et la méthodologie, des conceptions et des usages de la notion de diversité ethnoculturelle dans la formation initiale en enseignement sont mis en lumière. Je montre comment cette notion passe de contenu de formation à propriété de certains groupes de personnes. En élargissant davantage la problématique, je rends visibles certaines configurations des rapports sociaux de race en milieu universitaire. Ce faisant, je postule que la notion de diversité ethnoculturelle agit comme un voile sous lequel ces rapports sont à la fois dissimulés et réifiés. La discussion met en question certains de ses usages, en liant plus particulièrement la définition entérinée dans la politique de 1998 et d'autres, recueillies dans le cadre de ma recherche de maîtrise ${ }^{4}$.

2. En vertu du Règlement sur les autorisations d'enseigner (I-13.3), il est obligatoire d'avoir une autorisation d'enseigner pour dispenser l'éducation préscolaire et enseigner au primaire ou au secondaire à la formation générale et professionnelle. Depuis les réformes de 1992-1994 et 2001, la principale voie menant à son obtention est la réussite de l'un des 19 programmes de formation de premier cycle universitaire en enseignement de quatre ans, incluant également quatre stages obligatoires (700 heures ou plus).

3. Cette recherche a été réalisée avec le soutien financier du Conseil de recherches en sciences humaines.

4. Je remercie les arbitres et le comité de rédaction pour leurs commentaires ayant fortement contribué à la version définitive de cet article. 


\section{LES RAPPORTS SOCIAUX POUR ANALYSER L'INSTITUTION}

Le cadre conceptuel utilisé dans cet article rend explicite le renouvellement du regard posé sur l'institution éducative, entre la recherche de maîtrise et cette analyse secondaire. Dans leur sens le plus commun, les institutions sont plutôt appréhendées comme des structures figées (Tournay, 2011). Cette façon de les concevoir comporte le risque de les réifier, comme le postulent Peter Berger et Thomas Luckmann (1966/2012). Pour ces sociologues, la réification des phénomènes sociaux a comme danger la promotion d'une conception non dialectique de la relation entre ce que l'homme fait et ce qu'il pense, pouvant le conduire à ne plus avoir conscience que le monde social, même objectivé, a été façonné par les êtres humains et peut être recréé par ces derniers.

Une idée similaire oriente les analyses féministes matérialistes françaises, un courant constitué à partir de la méthode marxiste et des contenus du féminisme radical (Cervulle et Clair, 2017). Celles-ci ont comme ambition de démontrer la nature sociale, donc humaine et modifiable, de l'organisation sociale et des contraintes auxquelles la société prétend obéir (Delphy, 2013). Une analyse des rapports sociaux menée dans cette perspective confère la possibilité de «trouer le voile que les institutions jettent sur leur propre fonctionnement» (Delphy, 2013, p. 49) afin d'y glisser le regard.

Issu de la théorie marxiste, le rapport social est «une tension qui traverse le champ social et produit des enjeux matériels et idéels autour desquels se constituent des groupes aux intérêts antagoniques» (Kergoat, 2012, p. 17). Des travaux féministes ont montré, d'une part, l'existence des rapports sociaux de sexe qui, comme les rapports de classe, oppriment, exploitent et dominent et, d'autre part, que les rapports sociaux se reproduisent et se coproduisent mutuellement. Si la prédominance d'un discours universaliste caractérise une large part de la théorie féministe française (Maillé, 2014), Colette Guillaumin analyse les rapports sociaux de race dès la fin des années 1960.

Cette sociologue théorise l'idéologie raciste non pas comme une doctrine excluante, mais comme une forme d'intellectualisation par laquelle se construit et se maintient le pouvoir des «naturalisants» sur les naturalisés (Naudier et Soriano, 2010). L'idée de nature légitime les rapports de domination et d'appropriation d'êtres humains (Guillaumin, 1978a, 1978b). Le système esclavagiste était ainsi déjà constitué depuis environ un siècle lorsque les premières taxonomies raciales sont inventées (Guillaumin, 1977/2016). Cette idée de nature «revient toujours à dire que la Nature, cette nouvelle venue qui a pris la place des dieux, fixe les règles sociales" (Guillaumin, 1978b, p. 5).

Mais le tour de force de l'idée de nature perfectionnée avec les sciences modernes est de renverser le raisonnement. Des groupes «naturels» sont constitués sur la base de l'idéologie pour justifier une domination qui, elle, est matérielle: «l'idée de nature ne 
se réduit plus à une simple finalité sur la place des objets, mais elle prétend en outre que chacun d'entre eux, comme l'ensemble du groupe, est organisé intérieurement pour faire ce qu'il fait, pour être là où il est» (Guillaumin, 1978b, p. 5). Cette idée est étroitement liée à un renouvellement du système de marques, lesquelles deviennent l'origine même des rapports sociaux.

C'est donc toujours dans la perspective des rapports sociaux que j'utilise l'«idée de race» (Guillaumin, 1977/2016) ainsi résumée par Christine Delphy (2008) :

J'emploie le terme de race pour désigner non une réalité relevant de la génétique ou plus généralement de la nature, mais comme une construction sociale utilisant, comme le genre le fait pour le sexe, certaines caractéristiques morphologiques des individus pour constituer des groupes hiérarchiques. Pour être sociale, la race n'en est pas moins réelle, elle est même plus réelle qu'un phénomène physique qui existerait, mais serait méconnu socialement (p. 29).

Si ce mot est aujourd'hui tabou - «par censure, par prudence politique ou tout simplement par cynisme» (Guillaumin, 1981/2016, p. 208) -, le système de pensée raciste demeure aujourd'hui actif sous d'autres termes - comme la différence - qui héritent «de tout ce qui autrefois était porté par la notion de race elle-même: la spécificité de chaque groupe humain [naturalisé]» (Guillaumin, 1981/2016, p. 207). Sous ce terme ou un autre, l'idéologie naturaliste camoufle le rôle de la société, ce qui a pour effet de nous retirer «toute responsabilité de ce qui est, mais aussi toute possibilité d'inventer ce qui sera» (Delphy, 2013, p. 49). Le féminisme matérialiste offre l'appareillage conceptuel pour faire autrement.

\section{UNE ANALYSE SECONDAIRE POUR EXAMINER L'INSTITUTION SOUS UN AUTRE ANGLE}

Les sections qui suivent proposent une analyse secondaire des données de ma recherche de maîtrise, prolongeant et se distinguant de l'analyse issue de la collecte des données (Hakim, 1982, cité dans Dale, 1993). J'y présente un assemblage de fragments permettant de voir les rapports sociaux de race et, plus globalement, la circulation du pouvoir (Magar-Braeuner, 2017). La recherche a été réalisée à l'été 2013 sur trois sites se différenciant en ce qui a trait à l'établissement des personnes immigrantes sur leur territoire. La première (U1) se situe dans la région métropolitaine de Montréal, où s'établit la forte majorité des personnes immigrantes ${ }^{5}$. La seconde (U2) est localisée dans une région éloignée (Conseil supérieur de l'éducation, 2008), c'est-à-dire isolée des grands centres urbains. Le nombre de personnes

5. II s'agissait de $73,9 \%$ des personnes ayant immigré entre 2006 et 2015 , et toujours présentes sur le territoire au $1^{\mathrm{er}}$ janvier 2017 (Ministère de I'Immigration, de la Diversité et de I'Inclusion, 2017). 
immigrantes s'y établissant y est faible. La troisième université (U3) est située dans une région intermédiaire, où l'établissement de personnes immigrantes a été favorisé à partir des années 1990 par des politiques gouvernementales de régionalisation de l'immigration.

Pour documenter les processus d'institutionnalisation, les données ont été collectées du point de vue d'acteurs institutionnels. J'ai choisi de mener des entretiens semi-structurés auprès de neuf professeures ${ }^{6}$ ayant des tâches différentes dans l'institution. Dans chaque université, j'ai rencontré une professeure donnant au moins un cours abordant la diversité ethnoculturelle $(\mathrm{P})$, une professeure assumant la direction d'un programme de formation en enseignement (D) et la professeure assurant la «direction générale» de la faculté de l'université?.

D'une durée d'environ une heure, les entretiens portaient sur le travail des participantes en lien avec la diversité ethnoculturelle, les balises et les orientations institutionnelles à ce sujet au sein de leur université, et leur vision prospective de son institutionnalisation en formation initiale à l'enseignement. Chaque entretien a été transcrit et a fait l'objet d'une analyse qualitative thématique de type émergente site par site, puis de façon horizontale (Miles et Huberman, 2003). J'ai dégagé six facteurs ${ }^{8}$ pouvant avoir un effet sur l'institutionnalisation de la prise en compte de la diversité ethnoculturelle dans la formation initiale du personnel enseignant.

Les résultats présentés ci-après portent la marque de la méthode employée et de ses limites. Compte tenu de l'orientation institutionnelle initialement adoptée, la recherche s'appuie essentiellement sur les propos d'un nombre limité de professeures. Elle ne reflète donc pas le point de vue de la relève enseignante, pourtant au centre de la problématique étudiée, ni celui de l'ensemble du corps professoral. Par ailleurs, l'analyse secondaire se concentre principalement sur la dissimulation et la réification des rapports sociaux de race aux dépens de ce qui relève plutôt de la résistance et de la transformation de ces rapports.

6. Comme il n'y a que deux hommes sur l'ensemble des personnes interviewées, j'utilise le féminin pour me référer à celles-ci.

7. Par souci d'anonymat, le terme "faculté » est utilisé pour toutes les universités à l'étude sans égard à leur véritable structure organisationnelle (département ou unité des sciences de l'éducation).

8. Ces facteurs relèvent de dimensions individuelles, structurelles et conjoncturelles. En résumé, les facteurs individuels renvoient au poste occupé à l'université par les acteurs institutionnels et à leurs propres expériences relativement à la diversité ethnoculturelle à l'université ou à l'extérieur. Les facteurs structurels concernent l'organisation et le fonctionnement des universités auxquelles sont affiliées les professeures. Le premier facteur est l'institutionnalisation préalable de ce champ dans les programmes en formation en enseignement. Le second est l'existence de structures et d'occasions de concertation pour les membres du corps professoral. Les facteurs conjoncturels renvoient enfin au contexte local. Il s'agit, d'une part, de la présence historique et contemporaine de personnes immigrantes et racisées dans la région et, d'autre part, des expériences et des façons de concevoir cette présence par les acteurs de la formation initiale, dont les étudiantes et les étudiants (internes à l'université), et le personnel scolaire des écoles environnantes (externes à l'université) (Larochelle-Audet, 2014a, 2014b). 


\section{CONCEPTIONS ET USAGES DE LA DIVERSITÉ ETHNOCULTURELLE DANS LA FORMATION INITIALE EN ENSEIGNEMENT}

Dans la recherche, la diversité ethnoculturelle a été appréhendée comme un contenu de formation. Au fil des entretiens, une autre signification émerge. Elle passe de contenu de formation à propriété de certains groupes de personnes, les élèves, d'une part, et la relève enseignante, d'autre part ${ }^{9}$. Après avoir approfondi ces deux constats qui émergent à partir de l'appareillage conceptuel féministe matérialiste, le point de vue contrasté de professeures révèle d'autres fragments des rapports sociaux de race dans l'institution éducative.

\section{Un contenu de formation conditionnel aux publics}

Toutes les professeures interviewées jugent nécessaire de sensibiliser davantage les futurs membres du personnel enseignant à la diversité ethnoculturelle. Les avis divergent toutefois sur les moyens concrets de sa mise en œuvre, selon les responsabilités et les fonctions relatives aux postes occupés. Alors que les professeures spécialistes du champ estiment essentiel de formaliser davantage les contenus de formation, les professeures assumant des responsabilités de direction de programme ou de direction générale (professeures-directions) soutiennent qu'en l'absence d'une prescription formelle du ministère de l'Éducation, ceux-ci se trouvent en concurrence avec d'autres.

En tant que «responsables de la globalité de la qualité de la formation» (G2), elles doivent faire des choix. La diversité ethnoculturelle apparaît comme un contenu de formation supplémentaire parmi d'autres, voire comme «une autre forme [de diversité] dans laquelle malheureusement, on ne peut pas les préparer aussi bien qu'on voudrait» (G3). Les professeures-directions estiment que la responsabilité de cette formation relève en définitive des étudiantes et des étudiants eux-mêmes (p. ex., stages, travaux personnels, engagements bénévoles).

La pertinence, ou non, d'avoir un ou des cours sur la diversité ethnoculturelle dans les programmes est souvent justifiée par les besoins des étudiantes et des étudiants à court et à moyen terme pour l'enseignement. Cette tendance se reflète d'ailleurs dans le développement des nouveaux cours abordant cette question (Borri-Anadon et al., 2014), plus spécialisés selon les caractéristiques des publics scolaires. Les professeures-directions des deux universités situées en dehors de la métropole soutiennent ainsi qu'il est difficile de justifier tout ajout d'activités créditées s'il n’y a pas de demandes en ce sens formulées par la relève enseignante:

9. Compte tenu de la saillance de ce retournement de la problématique, cela a orienté ma thèse doctorale, qui sera publiée sous peu, ainsi qu'un article sur la transition vers l'emploi du personnel enseignant immigrant (Larochelle-Audet, 2017). 
C’est la réalité qui crée le besoin. Les barrières à ça sont là. Si jamais moi j'arrivais et que je disais: «On va créer un cours sur la différence culturelle demain matin", mon équipe va me dire: "Je pense que nos étudiants, ils sont confrontés à d'autres problématiques qu'il faudrait d'abord affronter » (D2).

Pour la professeure donnant les cours sur la diversité ethnoculturelle dans cette même université, comme pour celle travaillant dans une région où l'immigration est en croissance, la diversité ethnoculturelle ne constitue tout simplement pas une priorité institutionnelle. Elle demeure globalement appréhendée comme quelque chose d'étranger et de lointain ${ }^{10}$.

\section{Une propriété de certains groupes composant la relève enseignante}

Au cours des entretiens, les professeures introduisent une conception de la diversité ethnoculturelle excédant celle initialement imaginée pour la recherche. À l'exception d'une, toutes me parlent spontanément de l'accueil et de l'insertion en formation initiale en enseignement de personnes de groupes racisés. Alors que celles de l'université en région éloignée me présentent des initiatives d'accueil mises en place pour la relève enseignante autochtone, les professeures des universités situées à Montréal et dans une région visée par les politiques de régionalisation de l'immigration abordent sous différents aspects la présence de membres du personnel enseignant d'immigration récente dans leurs facultés. Elles abordent le sujet comme étant un «problème» dont la source varie selon les points de vue.

Traduisant leurs responsabilités, les professeures-directions de programmes ont une lecture similaire des problèmes attribués à l'arrivée de groupes d'enseignantes et d'enseignants immigrants. Elles soulignent en particulier certains de leurs comportements.

Je dirais que ça fait 5-6 ans qu'on accueille beaucoup d'étudiants d'immigration récente, mais avant les problèmes étaient beaucoup plus, comment dire, discrets. [...] Mais là, c'est des gens qui se manifestent plus aussi. Ils demandent des choses (D1).

Quand on a parti le programme [de maîtrise qualifiante], [...] on a trouvé que c'est devenu très populaire, très rapidement, avec des personnes déjà très frustrées par leur situation personnelle et qui avaient des attentes énormes envers notre système et ce que, nous, on pouvait leur fournir (D3).

10. Elle explique ceci: «Nous sommes des universités exportatrices d'enseignants. C'est-à-dire les enseignants que nous allons diplômer, ils [ne] vont pas tous travailler dans la région. Il y [en] a quelques-uns qui vont travailler dans la région où oui il y a peu d'immigration récente. [...] Mais alors, ces étudiants qui finissent ici, ils s'en vont enseigner où ce qu'il y a des postes. Et où ce qu'il y a des postes? La couronne nord, la couronne sud de Montréal, la région de l'0utaouais. Et là, oui, il y a des immigrants » (P2). 
Pour les trois professeures de l'université montréalaise, le "problème» se pose avec plus d'acuité dans le contexte des stages en milieu scolaire. Elles en offrent cependant des lectures différentes. D'un côté, les professeures-directions définissent le problème à partir de difficultés d'intégration associées notamment à un écart entre la culture du stage et celle des stagiaires:

Certains de nos stagiaires [...] peuvent parfois avoir de la difficulté à s'intégrer, à comprendre les codes culturels et la culture du milieu de stage où ils vont (G1).

L'autre point [problématique], c'est la gestion de classe. Parce que la gestion de classe, c'est fondé sur des valeurs. Ces valeurs-là sont issues de notre culture, et quand ils arrivent dans les classes, ils sont confrontés à un choc des valeurs et ils n'arrivent pas à intervenir de façon adéquate. C'est soit trop, trop drastique! Soit trop permissif quand ils veulent plaire (D1).

De l'autre côté, la professeure spécialiste de ce champ à cette même université entrevoit le problème sous un autre angle:

Le stage est souvent un enjeu pour nous aussi vis-à-vis des étudiants issus de l'immigration... et pas forcément à cause des étudiants. C'est une confrontation avec le milieu [scolaire]. Et que le milieu, des fois, ne sait pas comment réagir, et des fois, nous non plus. En fait, même assez souvent (P1).

Pour cette professeure, le problème relève d'abord du fonctionnement de l'institution éducative. Elle relate des histoires vécues par des stagiaires de son université pour montrer comment l'institution ferme les yeux sur des situations potentiellement discriminatoires:

Des stagiaires qui demandent à leur milieu de stage, avant le stage, est-ce que je suis en droit de porter tel habit traditionnel? [...] Ce n'est qu'une question pour permettre l'adaptation. Eh bien, l'enseignante associée rapporte ça au directeur, le directeur convoque l'avocat de la commission scolaire, et l'avocat de la commission scolaire est en rapport avec l'université en disant: «On va le prendre parce qu'on ne peut pas ne pas le prendre, parce qu'on va nous traiter de racistes, et on va nous poursuivre. Mais on ne veut plus d'étudiants de votre université (P1).

L'absence de régulation de telles situations ouvre de son point de vue la porte à des «problèmes de diversité ethnoculturelle mal vécue» (P1). 


\section{... ou un voile dissimulant les rapports sociaux de race?}

Les propos de cette professeure rendent visible l'autre côté de l'histoire (Smith, 2005). Son point de vue n'est probablement pas étranger à son expérience et à sa position dans les rapports sociaux de race, tel qu'elle me l'explique:

J'ai vécu plusieurs expériences interculturelles. Souvent, en tant d'ailleurs que... pas mal en tant qu'étrangère... plus qu'en tant que personne du pays d'accueil. Un peu comme je le suis maintenant. Sauf que, maintenant, je suis plus seulement une étrangère, je pense que j'ai pris ma place [rires] (P1).

Également immigrante, la professeure travaillant dans une université située en région éloignée m'ouvre à un point de vue similaire sur les rapports sociaux de race. Son récit montre comment des marques - accent, couleur de la peau, statut d'immigrant, etc. - deviennent l'origine de ses rapports (Guillaumin, 1981/2016) dans l'institution éducative. Elle qualifie sa première expérience d'enseignement d'un cours sur la diversité ethnoculturelle de «désastre». Un étudiant, soutenu par le groupe-classe, a ouvertement remis en question sa compétence:

La première année que je me suis... que j'ai donné le cours, ça a été très difficile. Je me suis même fait insulter dans le cours.

\section{À cause qu'ils ne se sentaient pas concernés?}

Non. À cause que moi, je ne devais pas enseigner parce que je n'étais pas Québécoise. [pause] «Je ne comprends pas votre accent.» Je me suis fait dire ça dans le cours (P2).

Cet événement «isolé, jusqu'à maintenant» (P2) l'a amenée par la suite à voir ce cours comme un défi personnel.

Elle m'explique avoir assumé cette charge d'enseignement sans être spécialiste du domaine. Elle a en effet répondu à la demande d'une direction de programme. Si elle en fait une blague, elle dit que sa nationalité d'origine (une marque) a pu motiver cette demande. Plusieurs années plus tard, elle demeure profondément déçue par le manque de soutien manifesté par la direction de la faculté à la suite de la situation vécue:

Sa réponse a été: «Ah, mais toi... Tu vas voir. Il faut comprendre... parce qu'ils ne connaissent pas... C'est la première fois qu'ils ont quelqu'un qui a un accent» (P2). 
Elle banalise cependant sa réaction en la situant dans le contexte général existant à la faculté, et plus largement au sein de l'université, où les préjugés sont courants. Elle me donne notamment cet exemple:

Lors d'une assemblée facultaire, un de nos collègues africains finit son évaluation quinquennale, et la question qu'on lui pose est: "Comment te sens-tu chez nous?» Alors lui - il a été très habile - il a demandé: "Chez vous... ça veut dire?» Et à ça, personne n'a réagi. Donc, c'est-à-dire qu'il y a «nous» et les «autres»... «Nous», on est les autres [Je souligne] (P2).

Avec son récit, cette professeure offre une illustration tangible des configurations du discours «Nous»/ «Eux» se manifestant dans le fonctionnement banal de l'institution (Dhume-Sonzogni, 2014; Potvin, 2008). Alors que les conceptions et les usages de la diversité ethnoculturelle dans les formations de la relève enseignante tendent à altériser et à extérioriser ces rapports - étant ceux des élèves ou de certains groupes de futurs enseignantes et enseignants -, ces situations montrent qu'ils se déroulent aussi à l'intérieur de l'institution, mais sont peu régulés.

\section{UNE QUESTION DE MOTS? UNE NOTION OCCULTANT LES RAPPORTS SOCIAUX DE RACE}

L'expression "diversité ethnoculturelle, linguistique et religieuse» se trouve dans la Politique d'intégration scolaire et d'éducation interculturelle (Ministère de l'Éducation, 1998). Elle désigne «une pluralité associée aux mouvements migratoires» (Ministère de l'Éducation, 1998, p. 3) s'étant ajoutée depuis la fin du siècle dernier à «la diversité historique de l'effectif scolaire québécois, dont les Canadiens français, les Canadiens anglais et les Amérindiens sont la manifestation» (Ministère de l'Éducation, 1998, p. 3). La "diversité de l'effectif scolaire» renvoie en définitive aux élèves «venant de tous les continents et dont les origines se sont diversifiées avec le temps ${ }^{11} »$ (Ministère de l'Éducation, 1998, p. 3).

Avec cette définition, la politique de 1998 institue un groupe composé d'un ensemble hétéroclite de personnes conçu en dehors des rapports de pouvoir entre les groupes et de l'histoire coloniale et d'esclavage du Québec (Potvin et Pilote, 2016; Trudel, 2004). Ce terme rend difficile la prise en compte des rapports sociaux de race, omettant notamment certains groupes constitués par ces rapports. C'est par exemple le cas des Afro-Canadiens établis sur le territoire depuis quatre siècles (Bessière, 2012), à la fois exclus de la diversité ethnoculturelle, religieuse et linguistique, et de la «diversité historique de l'effectif scolaire québécois». Ce terme permet difficilement de

11. Il est également précisé, dans la politique, que la "diversité de l'effectif scolaire se traduit sur le plan linguistique» (Ministère de l'Éducation, 1998, p. 3) et religieux, au regard du nombre de langues maternelles utilisées dans les établissements d'enseignement au Québec et de la représentation des grandes confessions religieuses. 
confronter le racisme systémique anti-noir profondément ancré dans les institutions canadiennes, comme l'a montré un rapport récent de l'Organisation des Nations Unies (2017).

Dans la recherche, la conception ayant émergé - la diversité ethnoculturelle comme propriété de certains groupes de personnes - s'avère également mouvante. Elle désigne quelquefois les personnes d'immigration récente, une catégorie juridico-politique regroupant les personnes arrivées il y a entre 5 et 10 ans selon les définitions. À d'autres moments, elle désigne plutôt les personnes ayant acquis leur qualification professionnelle à l'étranger. Mais parfois, ces mêmes termes sont utilisés pour désigner des personnes n'appartenant pas clairement à l'une ou l'autre de ces catégories. Ce terme rend difficile l'identification de ce qui fait problème.

Ce terme recouvre par ailleurs celui de différence, comme le montrent les définitions de la diversité avancées par les professeures ayant participé à la recherche ${ }^{12}$. La diversité est généralement conçue comme «une différence qu'on tourne bien [...] dont on essaie de tirer parti au profit des différences» (P1). Faisant référence au contexte scolaire, plusieurs professeures mettent l'accent sur le respect et l'ouverture aux différences: «La diversité, c'est [...] l'ouverture aux autres cultures, aux différences aussi, de toutes sortes» (D2). Cet élément fait écho à l'ouverture à la diversité ethnoculturelle, linguistique et religieuse largement valorisée dans la politique de 1998.

Derrière un vocabulaire positif, les notions de diversité et de différence dissimulent les inégalités sociales, les zones conflictuelles et les réalités que «l'on n'ose pas nommer», comme le racisme (Ahmed, 2009, 2012; Ricci, 2015). Elles peuvent également camoufler l'idée de nature et le système de marques la caractérisant (Guillaumin, 1977/2016). Une professeure évoque justement certaines marques morphologiques recouvertes par la notion de diversité: «il y a une certaine visibilité, ça peut être la race, l'ethnicité, la grandeur, un handicap physique, tout ça» (D3). Elle apparaît en partie comme étant quelque chose de «naturel»; une propriété des individus préexistant aux rapports sociaux (Dhume et Dukic, 2012). Si un discours critique des rapports de pouvoir ressort à d'autres moments dans les entretiens, il semble difficile pour les professeures de s'y référer dans le cadre circonscrit par la notion de diversité.

12. Afin de comprendre le sens y étant accordé par les professeures, l'une des premières questions posées dans le cadre de la recherche était: "De manière générale, comment définissez-vous la diversité?» (Larochelle-Audet, 2014a, p. 155). Le concept de diversité avait volontairement été dissocié des qualificatifs "ethnoculturelle», "linguistique» et "religieuse», de manière à ce que les professeures se sentent libres de l'aborder sous l'angle de leur choix. Elles étaient cependant informées de l'orientation de la recherche vers l'objet plus circonscrit de la diversité ethnoculturelle. Les extraits analysés peuvent être consultés dans Larochelle-Audet (2014a, p. 80-81). 


\section{PROSPECTIVES ET CONCLUSIONS: CHANGER DE POINT DE VUE}

L'appareillage conceptuel du courant féministe matérialiste a permis de mettre en question les conceptions et les usages de la notion de diversité ethnoculturelle. Avant d'être un contenu de formation, la diversité ethnoculturelle constitue une propriété de certains groupes, par exemple d'élèves et de personnel enseignant. C'est en partie leur présence dans l'institution éducative et les problématiques y étant associées qui justifient la prise en compte de certains contenus de formation. La notion en elle-même agit comme un voile qui efface le problème du racisme dans l'institution éducative et l'attribue aux «Autres» (Dhume, El Massioui et Sotto, 2016).

Bien que la recherche ait été initialement conduite du point de vue d'acteurs institutionnels, l'analyse secondaire effectuée montre la pertinence de partir d'autres points de vue. En effet, les fragments dévoilant avec le plus d'éloquence la circulation du pouvoir (Magar-Braeuner, 2017) ont été racontés par des professeures partageant à la fois une position similaire dans la division du travail - poste occupé, nombre d'années - et le système de stratification raciale (Olesen, 2011). Comme le montrent leurs récits, «des expériences partagées peuvent fournir des angles de vision similaires conduisant à un savoir collectif ou à un point de vue considéré comme essentiel à l'action" (Collins, 2000/2016, p. 442). En proposant un autre angle, les épistémologies $\mathrm{du}$ "point de vue» (stand point theory) introduisent une manière de "réorganiser les rapports sociaux régulant le savoir et sa production» (Smith, 2005, p. 29).

Comme le courant féministe matérialiste, ce type d'épistémologie ouvre à de nouvelles questions pour la recherche comme pour la formation des personnes travaillant dans l'institution éducative: comment s'assurer de ne pas participer à la reproduction de stéréotypes et, davantage encore, de catégories naturalisant les rapports sociaux? Comment favoriser l'émergence de «l'autre côté» de l'histoire, celle étant subsumée par les rapports sociaux et les processus institutionnels? Comment construire des savoirs alternatifs, libérateurs et émancipateurs? Ces questions proposent de rendre visibles les rapports sociaux, mais aussi de rendre possibles la résistance et la confrontation des divisions hiérarchiques par lesquelles certains groupes sont minorisés dans l'institution éducative. Prolongeant un appel déjà porté par Donatille Mujawamariya et Frédéric Saussez (2002), elles offrent une nouvelle perspective pour mener les réflexions sur ces enjeux dans la formation à l'enseignement, la profession enseignante et les finalités, et l'organisation du système éducatif au Canada, et en particulier dans les milieux. 


\section{Références bibliographiques}

AHMED, S. (2009). Embodying diversity: Problems and paradoxes for Black feminists. Race Ethnicity and Education, 12(1), 41-52.

AHMED, S. (2012). On being included: Racism and diversity in institutional life Durham, Royaume-Uni: Duke University Press.

BERGER, P. et LUCKMANN, T. (1966/2012). La construction sociale de la réalité. Paris, France: Armand Collin.

BESSIÈRE, A. (2012). La contribution des Noirs au Québec: quatre siècles d'une histoire partagée. Québec, Québec: Publications du Québec.

BORRI-ANADON, C., LAROCHELLE-AUDET, J., POTVIN, M. et Mc ANDREW, M. (2014). Bilan et enjeux de la formation initiale à la diversité ethnoculturelle, religieuse et linguistique en enseignement au Québec. Canadian Diversity / Diversité canadienne, 11(2), 59-64.

CERVULLE, M. et CLAIR, I. (2017). Lire entre les lignes: le féminisme matérialiste face au féminisme poststructuraliste. Comment s'en sortir? (4), 1-22. Repéré à https://commentsensortir.files.wordpress.com/2017/04/css4-2017-cervulle-clair-lireentre-les-lignes2.pdf

COLLINS, P.H. (2000/2016). La penséeféministenoire (2 éd., traduit par D. Lamoureux). Montréal, Québec: Remue-ménage.

Conseil supérieur de l'éducation. (2008). Rapport sur l'état des besoins en éducation 2006-2008. L'éducation en région éloignée: une responsabilité collective. Montréal, Québec: Ministère de l'Éducation, du Loisir et du Sport. Répéré à http://www. cse.gouv.qc.ca/fichiers/documents/publications/CEBE/50-0188.pdf

DALE, A. (1993). Le rôle de l'analyse secondaire dans la recherche en sciences sociales. Sociétés Contemporaines (14-15), 7-21. doi: https://doi.org/10.3406/ socco.1993.1124

DELPHY, C. (2008). Classer, dominer. Qui sont les «autres»? Paris, France: La Fabrique.

DELPHY, C. (2013). L'ennemi principal. 2. Penser le genre (3e éd.). Paris, France: Syllepse.

DHUME, F. et DUKIC, S. (2012). Orientation scolaire et inégalités de traitement selon «l'origine»: une synthèse critique des connaissances. Diversité VilleÉcole-Intégration, (167), 165-175. Repéré à https://halshs.archives-ouvertes.fr/ halshs-01421933/document 
DHUME, F., El MASSIOUI, N. et SOTTO, F. (2016). Former et enseigner sur la (non-) discrimination à l'école? Un enjeu politique incertain. Synthèse du rapport de recherche. Mars 2016. Paris, France: Institut social et coopératif de recherche appliquée (ISCRA) et Association Les Zégaux.

DHUME-SONZOGNI, F. (2014). Entre l'école et l'entreprise: la discrimination en stage. Une sociologie publique de l'ethnicisation des frontières scolaires. Aix-enProvence, France: Presses universitaires de Provence.

GUILLAUMIN, C. (1977/2016). Race et Nature. Système des marques, idée de groupe naturel et rapports sociaux (article initialement paru en 1977 dans Pluriel, (11), 39-55). Dans C. Guillaumin (dir.), Sexe, race et pratique du pouvoir: l'idée de nature (p. 165-187). Donnemarie-Dontilly, France: iXe.

GUILLAUMIN, C. (1978a). Pratique du pouvoir et idée de Nature (1) L'appropriation des femmes. Questions Féministes, (2), 5-30.

GUILLAUMIN, C. (1978b). Pratique du pouvoir et idée de Nature (2) Le discours de la Nature. Questions Féministes, (3), 5-28.

GUILLAUMIN, C. (1981/2016). «Je sais bien, mais quand même» ou les avatars de la notion de race (article initialement paru en 1981 dans Le Genre humain, (1), 55-65). Dans C. Guillaumin (dir.), Sexe, race et pratique du pouvoir: l'idée de nature (p. 165-187). Donnemarie-Dontilly, France: iXe.

KERGOAT, D. (2012). Se battre, disent-elles. Paris, France: La Dispute.

LAROCHELLE-AUDET, J. (2014a). Acteurs du milieu universitaire et formation initiale à la diversité ethnoculturelle en enseignement: entre engagement et institutionnalisation. (Mémoire de maîtrise, Université du Québec à Montréal, Canada). Repéré à http://www.archipel.uqam.ca/6572

LAROCHELLE-AUDET, J. (2014b). La diversité ethnoculturelle dans la formation initiale au Québec. Diversité, 177, 155-160.

LAROCHELLE-AUDET, J. (2017). La transition vers l'emploi des enseignants immigrants: nouvelles perspectives de recherches. Initio, revue sur l'éducation et la vie au travail, (6, printemps 2017), 75-94.

LAROCHELLE-AUDET, J., BORRI-ANADON, C., Mc ANDREW, M. et POTVIN, M. (2013). La formation initiale du personnel scolaire sur la diversité ethnoculturelle, religieuse et linguistique dans les universités québécoises: portrait quantitatif et qualitatif. Rapport de recherche du Centre d'études ethniques des universités montréalaises/Chaire de recherche du Canada sur l'éducation et les rapports ethniques. Montréal, Québec: Ministère de l'Éducation, du Loisir et du Sport. 
LAROCHELLE-AUDET, J., BORRI-ANADON, C. et POTVIN, M. (2016). La formation interculturelle et inclusive du personnel enseignant: conceptualisation et opérationnalisation de compétences professionnelles. Éducationetfrancophonie, XLIV(2), 172-195.

LAROCHELLE-AUDET, J., MAGNAN, M.-O., POTVIN, M. et D'ARRISSO, D. (2018). Le personnel scolaire et la diversité ethnoculturelle au Québec: regard sur les prescriptions normatives. Dans C. Borri-Anadon, G. Gonçalves, S. Hirsch et J. di Paula Queiroz Odinino (dir.), La formation des éducateurs en contexte de diversité ethnoculturelle: une perspective comparative Québec-Brésil (p. 106-123). Blue Mounds, WI: Deep Education Press.

MAGAR-BRAEUNER, J. (2017). Enquête sur la microphysique du pouvoir à l'école: actualisation, imbrication des rapports de domination et modalités d'une pédagogie émancipatrice (Thèse de doctorat, Université du Québec à Montréal, Canada, et Université de Paris Vincennes Saint-Denis, France).

MAILLÉ, C. (2014). Approche intersectionnelle, théorie postcoloniale et questions de différence dans les féminismes anglo-saxons et francophones. Politique et Sociétés, 33(1), 41-60.

MILES, M. B. et HUBERMAN, M. A. (2003). Analyse des données qualitatives (2e éd.). Bruxelles, Belgique: De Boeck.

Ministère de l'Éducation. (1998). Politique d'intégration scolaire et d'éducation interculturelle. Québec, Québec: Gouvernement du Québec.

Ministère de l'Éducation. (2001a). La formation à l'enseignement: les orientations et les compétences professionnelles. Québec, Québec: Gouvernement du Québec.

Ministère de l'Éducation. (2001b). La formation à l'enseignement professionnel: les orientations et les compétences professionnelles. Québec, Québec: Gouvernement du Québec.

Ministère de l'Immigration, de la Diversité et de l'Inclusion (2017). Fiche synthèse sur l'immigration au Québec - 2016. Gouvernement du Québec. Repéré à http:// www.midi.gouv.qc.ca/publications/fr/recherches-statistiques/FICHE_syn_an2016.pdf

MOLDOVEANU, M. et MUJAWAMARIYA, D. (2007). L'éducation multiculturelle dans la formation initiale des enseignants: des politiques aux pratiques. McGill Journal of Education/Revue des sciences de l'éducation de McGill, 42(1), 31-46.

MUJAWAMARIYA, D. (dir.). (2006). L'éducation multiculturelle dans la formation des enseignants au Canada. Dilemmes et défis. New York, NY: Peter Lang. 
MUJAWAMARIYA, D. et MOLDOVEANU, M. (2006). Les paradoxes du multiculturalisme: de la préparation des futurs enseignants dans les grandes universités canadiennes. Formation et pratiques d'enseignants en question, 4, 139-161.

MUJAWAMARIYA, D. et SAUSSEZ, F. (2002). Conclusion. Dans D. Mujawamariya (dir.), L'intégration des minorités visibles et ethnoculturelles dans la profession enseignante: récits d'expériences, enjeux et perspectives. Outremont, Québec: Logiques.

NAUDIER, D. et SORIANO, É. (2010). Colette Guillaumin. La race, le sexe et les vertus de l'analogie. Cahiers du Genre, 48(1), 193-214.

OLESEN, V. (2011). Feminist qualitative research in the millenium's first decade: Developments, challenges, prospects. Dans N. K. Denzin \& Y. S. Lincoln (dir.), Handbook of qualitative research ( $4^{\mathrm{e}}$ éd., p. 129-146). Thousand Oaks, CA: Sage.

Organisation des Nations Unies. (2017). Le racisme, la discrimination raciale, la xénophobie et l'intolérance qui est associée - suivi et application de la Déclaration et du Programme d'action de Durban. Assemblée générale des Nations Unies: rapport du Groupe de travail d'experts sur les personnes d'ascendance africaine sur sa mission au Canada, A/HRC/36/60/Add.1, en date du 16 août 2017. Repéré à http://undocs.org

POTVIN, M. (2008). Crise des accommodements raisonnables: une fiction médiatique? Outremont, Québec: Athéna.

POTVIN, M. (2016). Interethnic relations and racism in Quebec. Dans S. Gervais, C. Kirkey et J. Rudy (dir.), Quebec questions: Quebec studies for the $21^{\text {st }}$ century (2 éd., p. 271-296). Toronto, Ontario: Oxford University Press.

POTVIN, M., BORRI-ANADON, C., LAROCHELLE-AUDET, J., ARMAND, F., Cividini, M., Chastenay, M.-H. (2015). Rapport sur la prise en compte de la diversité ethnoculturelle, religieuse et linguistique dans les orientations et compétences professionnelles en formation à l'enseignement. Montréal, Québec: Groupe de travail interuniversitaire sur les compétences interculturelles et inclusives en éducation, Observatoire sur la formation à la diversité et l'équité. Repéré à http://collections.banq.qc.ca/ark:/52327/bs2482627

POTVIN, M. et PILOTE, A. (2016). Les rapports ethniques et les processus d'exclusion. Dans M. Potvin, M.-O. Magnan et J. Larochelle-Audet (dir.), La diversité ethnoculturelle, religieuse et linguistique en éducation au Québec. Théorie et pratique (p. 79-98). Montréal, Québec: Fides Éducation. 
RICCI, S. (2015). Quand le sourire de la diversité cache les rapports de domination. Dans N. Hamrouni et C. Maillé (dir.), Le sujet du féminisme est-il blanc? Femmes racisées et recherche féministe (p. 175-193). Montréal, Québec: Remue-ménage.

SMITH, D. E. (2005). Institutional ethnography: A sociology for people. Toronto, Ontario: AltaMira Press.

TOURNAY, V. (2011). Sociologie des institutions. Paris, France: Presses universitaires de France.

TRUDEL, M. (2004). Deux siècles d'esclavage au Québec. Montréal, Québec: Hurtubise $\mathrm{HMH}$. 